\title{
Naltrexone Alters Subjective and Psychomotor Responses to Alcohol in Heavy Drinking Subjects
}

\author{
Mary E. McCaul, Ph.D., Gary S. Wand, M.D., Thomas Eissenberg, Ph.D., \\ Charles A. Rohde, Ph.D., and Lawrence J. Cheskin, M.D.
}

Preclinical studies support endogenous opioid system involvement in alcohol reinforcement and consumption; however, recent clinical trials and human laboratory studies have provided mixed findings of the effects of naltrexone (a non-selective opioid antagonist) on alcohol responses. This study used a within-subject design $(\mathrm{n}=23)$ to investigate naltrexone effects $(0,50$ and $100 \mathrm{mg} q d)$ on subjective and psychomotor responses to alcohol (none, moderate, high) in heavy drinkers. Before alcohol administration, subjects reported decreased desire to drink alcohol when maintained on $50 \mathrm{mg}$ compared with placebo naltrexone. Following alcohol administration, active naltrexone significantly increased subjective ratings of sedative, and unpleasant/sick effects and decreased ratings of liking, best effects and desire to drink. Naltrexone generally did not alter subjective or objective indicators of drunkenness. Finally, high doses of naltrexone and alcohol interacted to produce the greatest decreases in liking and best effects. Findings support the role of endogenous opioids as determinants of alcohol's effects and suggest that naltrexone may be particularly clinically useful in those treatment patients who continue to drink heavily. [Neuropsychopharmacology 22:480-492, 2000] (c) 2000 American College of

Neuropsychopharmacology. Published by Elsevier Science Inc.
KEY WORDS: Alcoholism; Naltrexone; Opioid; Subjective responses; Psychomotor responses

Over the last decade, preclinical and clinical research have convincingly demonstrated the involvement of the endogenous opioid system in alcohol reinforcement and consumption. First, it is clear that opioid agonist and antagonist administration alters alcohol consump-

From the Johns Hopkins University School of Medicine (MEM, GSW, LJC), Virginia Commonwealth University (TE), Richmond, Virginia; and Johns Hopkins University School of Public Health (CAR), Baltimore, Maryland.

Address correspondence to: Mary E. McCaul, Ph.D., Department of Psychiatry and Behavioral Sciences, Johns Hopkins University School of Medicine, 911 N. Broadway, Baltimore, MD 21205. Tel.: 410-955-5439; Fax: 410-955-4769.

Received December 30, 1998; revised October 25, 1999; accepted November 5, 1999. tion (Czirr et al. 1987; Hubbell et al. 1986; Reid et al. 1991). For example, animal studies have demonstrated slowed acquisition of alcohol self-administration (Phillips et al. 1997) and decreased alcohol consumption following administration of the non-selective opioid receptor antagonists naloxone (Froehlich et al. 1990; Hubbell et al. 1986; Hyytia and Sinclair 1993 ; Reid et al. 1991; Weiss et al. 1990), naltrexone (Altshuler et al. 1980; Boyle et al. 1998; Davidson and Amit 1997 ; Myers et al. 1986; Reid et al. 1996; Volpicelli et al. 1986), and nalmefene (Hubbell et al. 1991). The second line of evidence for functional involvement of the opioid system in mediating alcohol drinking behavior comes from neurobiological studies which indicate that alcohol alters opioid peptide systems. Acute alcohol administration increases endorphin and enkephalin gene expression in discrete brain regions of rodents, increases opioid peptide release from both brain and pituitary of 
rodents in vitro, and increases opioid peptide release from the pituitary of rodents and humans in vivo (Gianoulakis 1990 ; Gianoulakis et al. 1996; Li et al. 1996). The third line of evidence for functional involvement of the opioid system in mediating alcohol drinking behavior is derived from genetic studies which indicate that a genetic predisposition toward alcohol drinking is accompanied by altered responsiveness of the opioid system at baseline and in the presence of alcohol in both rodents and humans (Gianoulakis et al. 1996; KrishnanSarin et al. 1995; Li et al. 1996; Wand et al. 1998). For instance, alcohol consumption induces a more robust increase in plasma beta-endorphin in subjects with a positive family history of alcoholism compared to family history negative subjects (Gianoulakis et al. 1996).

These findings involving the endogenous opioid system in alcoholism have led to several recent clinical trials examining the effectiveness of opioid antagonists for the treatment of recently abstinent alcohol-dependent subjects. Two 12-week, double-blind clinical trials (O'Malley et al. 1992; Volpicelli et al. 1992) reported the effectiveness of naltrexone in combination with psychosocial treatment participation in decreasing relapse and alcohol drinking days. Most striking was a reduction of full-blown relapse (consumption of five or more drinks per occasion) among those patients who resumed drinking, with the risk of relapse among placebotreated patients approximately twice that of naltrexonetreated patients. Findings suggest that the effectiveness of naltrexone in preventing alcohol relapse may stem from a decrease in the subjective experience of alcohol "high" or intoxication following alcohol consumption among naltrexone-treated compared to placebo-treated patients (O'Malley et al. 1996; Volpicelli et al. 1995); however, these findings are confounded by lower levels of alcohol consumption per drinking episode among naltrexone patients.

Human behavioral pharmacology laboratory studies can provide sensitive tests of potential pharmacotherapies for persons with alcohol or drug use disorders. Generally, the interaction of a given medication with its target drug is more easily detected in the laboratory than in the clinic (Bigelow and Walsh 1998); however, to date, findings of human laboratory research examining the interaction of naltrexone and alcohol have been equivocal. Doty and colleagues (Doty and de Wit 1995 ; Doty et al. 1997) have reported no effects of naltrexone pretreatment ( 25 or $50 \mathrm{mg}$ ) on subjective responses following a range of alcohol doses (placebo, $0.25 \mathrm{~g} / \mathrm{kg}$, $0.50 \mathrm{~g} / \mathrm{kg}, 0.75 \mathrm{~g} / \mathrm{kg}$ ) in social drinkers. In contrast, Swift and colleagues (Swift et al. 1994) observed reduced stimulant effects and increased sedative effects of alcohol following acute naltrexone $(50 \mathrm{mg})$ as compared with placebo administration in social drinkers. Also, chronic naltrexone administration $(50 \mathrm{mg}$ ) has been reported to increase latency to drink alcohol in so- cial drinkers in a naturalistic bar setting, although no differences were observed in self-reported urge to drink or liking, stimulant or sedative effects, number of drinks ordered, or latency to finish the beverage (Davidson and Amit 1997). Finally, recent findings have suggested the potential role of family history of alcoholism as a determinant of naltrexone effects on alcohol responses (King et al. 1997). Naltrexone (50 mg) decreased alcohol's stimulant effects on the rising BAL limb in family history positive (FHP) but not family history negative subjects. In both family history groups, naltrexone decreased vigor and increased fatigue, tension and confusion scores on the Profile of Mood States, with FHP subjects evidencing increased confusion on the ascending BAL limb following naltrexone.

The present human laboratory study has a number of design strengths. First, this project used a completely within-subject design, thus maximizing statistical sensitivity. Second, a range of naltrexone doses $(0,50$ and $100 \mathrm{mg}$ qd) were examined under chronic dosing procedures. Within each naltrexone dose condition, a range of alcohol doses (no, moderate and high) were studied. This dosing procedure allowed for the examination of both direct and interactive effects of alcohol and naltrexone on responses. Finally, a broad spectrum of measures including subjective and psychomotor responses were obtained repeatedly before and for an extended period after alcohol ingestion within each session, allowing in-depth assessment of time course of effects.

\section{METHODS}

\section{Subjects}

Potential study participants $(\mathrm{N}=99)$ were recruited with newspaper advertisements and word of mouth. Persons aged 25-60 with moderate to heavy alcohol use (an average of five drinks per occasion or five drinking days per week), low levels of associated problems (MAST score < 5; (Selzer 1971), and no acute or chronic health problems were eligible to participate in a clinical interview and medical examination to determine study eligibility. Exclusion criteria included: current use of any prescription medication, chronic health problems, liver enzyme levels over twice normal limits, pregnancy, a major Axis I diagnosis on the Structured Clinical Interview for DSM-IIIR (Spitzer and Williams 1987), including alcohol or drug abuse/dependence. Seventytwo individuals were determined to be ineligible for study participation due to a diagnosis of alcohol abuse/ dependence and/or medical or psychiatric exclusions.

Twenty-seven subjects participated in one or more sessions of the laboratory study. Of these, four were subsequently excluded from data analysis: one was withdrawn from the study prematurely due to health concerns unrelated to study medication, one subject 
had excessive missing data, and two were disqualified due to illicit drug use detected during routine urine screening prior to study sessions.

Table 1 summarizes the demographic and substance use characteristics of the twenty-three subjects whose data are presented in the present report. Four were female and 11 were of minority ethnic/racial status. Subjects averaged 37 years old and all subjects had a high school education or greater. Two-thirds were employed at the time of their research participation and almost all had either never been married or were separated or divorced. Subjects reported drinking an average of almost five standard drinks per drinking occasion, consumed alcohol on an average of 13 days per month, and had been drinking alcohol for an average of 19 years. Slightly more than half of the subjects smoked tobacco cigarettes and they averaged about three-quarters of a pack per day. Almost half reported occasional marijuana use and a quarter of the sample also used cocaine occasionally.

Subjects were informed that the study was intended to determine how drugs from different pharmacologic classes modify alcohol's effects. All potential subjects signed an institution-approved informed consent document prior to any research participation and were paid for their time in the study.

\section{Procedures}

During their first visit, subjects participated in the clinical interview and medical screen. Eligible subjects then received massed practice of ten trials on each of the

Table 1. Demographic and Recent Substance Use Characteristics $(\mathrm{N}=23)$

\begin{tabular}{lcc}
\hline Characteristics & Mean \pm SD & Range \\
\hline Age (yrs) & $38.3(7.7)$ & $28.5-55.5$ \\
Education (yrs) & $13.1(2.5)$ & $10-20$ \\
Females (\%) & 17 & \\
Race/ethnicity (\%): & 48 & \\
$\quad$ White & 48 & \\
$\quad$ African-American & 4 & \\
$\quad$ Hispanic & 65 & \\
Employed (\%) & & \\
Marital status (\%) & 58 & $6-10$ \\
$\quad$ Never & 4 & $7-33$ \\
$\quad$ Married & 48 & \\
$\quad$ Separated/divorced & & \\
Alcohol use: & $4.6(2.0)$ & \\
$\quad$ Drinks/occasion & $12.0(4.0)$ & \\
$\quad$ Days/month & $19.7(7.4)$ & \\
$\quad$ Years of use & & \\
Tobacco use: & 57 & \\
$\quad \%$ Smokers & $15.2(5.4)$ & \\
$\quad$ Cigarettes/day & 39 & \\
Marijuana users (\%) & 26 & \\
Cocaine users (\%) & & \\
\hline
\end{tabular}

psychomotor tasks (described below) to attain a stable performance baseline prior to starting the experiment. Subjective report items also were reviewed to familiarize subjects with these measures.

All study sessions were conducted at the General Clinical Research Center (GCRC) at the Johns Hopkins Bayview Medical Center. Subjects participated in the research over a 6-week period. Each of three naltrexone doses was administered over an 8-day inpatient stay on the GCRC. There was a one-week outpatient wash-out period between each naltrexone dose period.

Within each naltrexone dose, GCRC admission was on Friday; the naltrexone dose scheduled for that week was administered daily at $8 \mathrm{pm}$ for 7 days starting on the day of admission. Over the course of the 8-day inpatient stay, subjects participated in alcohol challenge sessions on Monday, Wednesday and Friday. Subjects were discharged from the GCRC on Saturday morning.

On each alcohol challenge session day, subjects were provided with a standard (no caffeine) breakfast at 7:30 A.M. and a snack at 10:00 A.M.. Neither cigarette smoking nor additional food or drink were permitted between 10:00 A.M. and 3:30 P.M.. Sessions began with the insertion of a heparinized catheter in a non-dominant forearm vein approximately 90 minutes prior to alcohol administration. Subjects remained in a quiet room during testing, and remained seated except as required for performance measures.

\section{Drug Preparation and Administration}

All subjects participated in nine sessions according to a completely within-subject, randomized block design. Each subject received the three naltrexone dose conditions (0,50 and $100 \mathrm{mg} \mathrm{po})$ in random order. Each daily naltrexone dose was prepackaged and individually labeled and dated using $50 \mathrm{mg}$ naltrexone tablets and matching placebo (DuPont Pharma). Subjects ingested naltrexone under observation by the GCRC nursing staff at 8 p.m. daily.

Each of the three alcohol dose conditions (no, moderate, high) was presented in random order within each naltrexone dose condition. Alcohol drinks were prepared by mixing the appropriate amount of pure ethanol (minus $3 \mathrm{ml}$ which was added just prior to administration, see below) with juice to a volume of 16 ounces. The drink was separated into three equal amounts; subjects had five minutes to drink each glass (McCaul et al. 1990). In this report, "time 0 " and "alcohol administration" both refer to the end of this 15-minute administration procedure. In order to conceal the alcohol content of the drink, a wrist band soaked in ethanol was placed around the glass to deliver an alcohol odor and $1 \mathrm{ml}$ of ethanol was floated on top of each glass to deliver an ethanol taste. Alcohol dose levels were 0.0, 0.6 and 1.2 
$\mathrm{g} / \mathrm{kg}$ for the first four subjects to complete the study. Dose levels were reduced to $0.0,0.5$ and $1.0 \mathrm{~g} / \mathrm{kg}$ for the remainder of the study participants due to excessive intoxication following the initial high alcohol dose level. For analysis, these dose conditions have been combined into no, moderate and high alcohol doses. GCRC nurses, the study research assistant and subjects were blind to all ethanol and naltrexone doses.

\section{Dependent Measures}

During each alcohol challenge session, subjects participated in repeated administrations of a comprehensive test battery that included subjective reports, autonomic data collection, a blood draw through the indwelling catheter and performance tasks in this order. Relative to alcohol administration (time 0 ), blood samples for neuroendocrine and blood alcohol determinations were drawn at $-30,-15,0,15,30,60,90,120,150$ and $180 \mathrm{~min}-$ utes. Recordings of heart rate, blood pressure, skin temperature and conductance began 60 minutes prior to alcohol administration and were continuous until 180 minutes after alcohol administration. Subjective and performance measures were obtained at $-60,0,15$, $30,60,90,120,150,180,240,300,360,420$, and $480 \mathrm{~min}-$ utes. Neuroendocrine and physiological measures are not included in the present report.

Subjective measures were displayed using an Apple IIE microcomputer and completed by subjects using a joystick that moved a cursor on the screen to mark the appropriate response. Measures included visual analog scales (VAS), the Subjective High Assessment Scale (SHAS) (Schuckit 1980), and the short-form of the Addiction Research Center Inventory (ARCI; (Haertzen 1974). The visual analog scales used a horizontal line anchored with "Not at all" on the left and "Extremely" on the right. The four VAS items included the questions: "How high do you feel right now?", "How sleepy do you feel?", "How badly would you like an alcohol drink right now?", and "How do you like the effects of the capsule/beverage you received?"

The Subjective High Assessment Scale consists of fifteen brief descriptors of alcohol effects, including uncomfortable, high, clumsy, muddled or confused, slurred speech, dizzy, nauseated, drunk or intoxicated, sleepy, distorted sense of time, effects of alcohol or drug, difficulty concentrating, feelings of floating, the worst I've ever felt, and the best I've ever felt. Subjects were instructed to respond for how they felt "right now" using a horizontal line anchored by "Normal" on the left and "Extremely" on the right.

The short-form of the Pentobarbitol-Chlorpromazine-Alcohol Group (PCAG) subscale of the Addiction Research Inventory consisted of 15 items sensitive to sedative drug effects.

Performance measures consisted of a balance measure, a reaction time task and two tests of memory. Balance was measured by asking subjects to stand upright on one foot with eyes closed and arms extended to the side at shoulder height. Each trial lasted 30 seconds or until the subject touched the raised foot to the ground. Subjects completed two trials on each foot during each data collection period, thus the maximum score was $60 \mathrm{sec}-$ onds measured separately for the right and left foot.

Reaction time was assessed using the circular lights apparatus which consists of 16 lights illuminated in random order and arranged circularly around a $54-\mathrm{cm}$ diameter. When a button adjacent to each light was pressed, the light extinguished and the next bulb was lit. Reaction time was determined by the number of lights that subjects extinguished in a 60 -second period.

The computerized version of the Digit-Symbol Substitution Test is described elsewhere (McLeod et al. 1982). In response to random digits (1-9) that appeared in the center of the video screen, subjects pressed button positions on a numeric keypad to reproduce the geometric pattern associated with that digit by using the digit-symbol code displayed continuously at the top of the video screen. The scores were the number attempted and the number of correct substitutions during a 90-sec trial.

A computerized numeric recall task assessed shortterm memory. During each trial, an 8-digit number was displayed on the computer screen. First, subjects entered the number while it was still displayed on the screen. If a subject entered the number incorrectly while it was still displayed, the trial was discontinued and a different 8-digit number was presented. If the subject entered the number correctly, the number then disappeared from the screen and, either immediately ( 5 trials) or after a 8-second delay (five trials), subjects entered the number from memory. The task continued until the subject had correctly entered ten 8-digit numbers during the first component of the task or 25 incorrect attempts were made. Scores included the total number of trials attempted and the number of digits correctly reproduced during the recall component of the task.

\section{Data Analysis}

Statistical analysis was performed using STATA 5.0 and its GEE module. Analyses were based on a longitudinal analysis with subject as a random effect. Variables included alcohol dose, naltrexone dose and time. For each measure, baseline levels were first examined as a function of alcohol and naltrexone dose levels. When significant baseline effects were observed, baseline values of the variable were entered as covariates in subsequent analyses. Initial analyses examined the independent and interaction effects of each active alcohol and naltrexone dose compared to placebo. When the interac- 
tions were not significant, the final model was simplified to examine only the independent effects of alcohol and naltrexone. For each response variable, the traditional significance level of $p=.05$ was adjusted using Bonferroni's method to $p=.01$ to compensate for analyses examining two main effects of alcohol, two main effects of naltrexone, and time. Significance levels for alcohol $\mathrm{X}$ naltrexone interactions were set at $p=.05$ since these interactions were the primary scientific focus of the research.

\section{RESULTS}

\section{Subjective Effects}

Desire to Drink. At baseline prior to alcohol administration, subjects maintained on $50 \mathrm{mg}$ naltrexone reported a decreased desire to drink alcohol compared to subjects maintained on placebo naltrexone $(p<.01)$. There also was a trend for decreased desire to drink at baseline for the $100 \mathrm{mg}$ compared to placebo naltrexone condition $(p<.10)$. There was a significant effect of baseline ratings of desire to drink on ratings following alcohol administration $(p<.001)$; that is, the higher a subject's baseline report, the higher the subject's postingestion rating.
Ingestion of the moderate or high alcohol dose did not alter desire to drink when subjects were maintained on placebo naltrexone. In contrast, subjects maintained on $100 \mathrm{mg}$ naltrexone reported significantly less desire to drink following alcohol administration compared to placebo naltrexone (Table 2). The moderate dose of naltrexone did not affect self-reported desire to drink following alcohol administration.

Active compared to placebo naltrexone disrupted alcohol consumption during the drink ingestion period. During at least one of their nine sessions, twelve of the 23 subjects either refused to drink a portion of the alcohol beverage $(\mathrm{N}=5)$ and/or drank the beverage at a slower pace than the 15-minute study procedure allowed ( $\mathrm{N}=9 ; \mathrm{X}=27$ minutes; range: $20-45$ minutes). Eleven of the 12 subjects experienced the first disruption of their alcohol ingestion during an active naltrexone session and only one subject during a placebo naltrexone session $(Z=-5.21, p<.0001)$.

Best/Like Effects. All three alcohol doses increased subjects' reports of "like effects of capsule/beverage" and "best I've ever felt" over baseline responses prior to drink ingestion. Subjects' analog ratings of "like effects" were comparable across the three alcohol doses. Subjects' reports of "best I've ever felt" were somewhat

Table 2. Separate and Interactive Effects of Naltrexone and Alcohol on Subjective Responses. Arrows Indicate Direction of Effect

\begin{tabular}{|c|c|c|c|c|c|}
\hline Subjective Effects & Nx 0 vs 50 & Nx 0 vs 100 & Alc 0 vs Mod & Alc 0 vs High & Interactions \\
\hline DESIRE TO DRINK & & $\downarrow^{b}$ & & & \\
\hline BEST & $\downarrow^{c}$ & & $\downarrow^{c}$ & & $\uparrow 50-\mathrm{M}^{a}, \downarrow 100-\mathrm{H}^{b}$ \\
\hline LIKING & $\downarrow c$ & & & & $\uparrow 50-\mathrm{M}^{a}, \downarrow 100-\mathrm{H}^{a}$ \\
\hline \multicolumn{6}{|l|}{ Sick/unpleasant effects } \\
\hline Uncomfortable & $\uparrow c$ & $\uparrow b$ & $\uparrow c$ & $\uparrow c$ & \\
\hline Nauseated & $\uparrow_{c}$ & $\uparrow c$ & $\uparrow c$ & $\uparrow c$ & \\
\hline Worst I've felt & $\uparrow_{c}$ & & $\uparrow_{c}$ & $\uparrow_{c}$ & \\
\hline \multicolumn{6}{|l|}{ Sedative effects } \\
\hline Clumsy & $\uparrow b$ & $\uparrow^{c}$ & $\uparrow c$ & $\uparrow c$ & \\
\hline Confused & $\uparrow b$ & $\uparrow c$ & $\uparrow_{c}$ & $\uparrow_{c}$ & \\
\hline Slurred speech & & $\uparrow b$ & $\uparrow c$ & $\uparrow c$ & \\
\hline Sleepy & & $\uparrow b$ & $\uparrow c$ & $\uparrow c$ & \\
\hline Trouble concentrate & $\uparrow b$ & & $\uparrow c$ & $\uparrow c$ & \\
\hline Floating & & & $\uparrow_{c}$ & $\uparrow_{c}$ & $\downarrow 100-\mathrm{H}^{a}$ \\
\hline Dizzy & $\uparrow c$ & $\uparrow b$ & $\uparrow c$ & $\uparrow c$ & \\
\hline Time distorted & & $\uparrow b$ & $\uparrow c$ & $\uparrow_{c}^{c}$ & $\downarrow 100-\mathrm{M}^{b}, 100-\mathrm{H}^{b}$ \\
\hline PCAG SCALE & & & $\uparrow c$ & $\uparrow c$ & $\downarrow 100-\mathrm{M}^{b}$ \\
\hline \multicolumn{6}{|l|}{ High/intoxicated } \\
\hline High & & & $\uparrow c$ & $\uparrow c$ & $\uparrow 50-\mathrm{H}^{a}$ \\
\hline Drunk & & & $\uparrow c$ & $\uparrow_{c}$ & \\
\hline Feel effects & $\uparrow b$ & & $\uparrow c$ & $\uparrow c$ & \\
\hline
\end{tabular}

$a=p \leqslant .05$.

$b=p \leqslant .01$.

$c=p \leqslant .001$.

$\mathrm{M}=$ moderate alcohol dose

$\mathrm{H}=$ high alcohol dose

$50=$ naltrexone $50 \mathrm{mg}$

$100=$ naltrexone $100 \mathrm{mg}$ 
lower following the moderate alcohol dose in comparison to high dose or placebo alcohol.

There was a main effect of $50 \mathrm{mg}$ naltrexone on the items of "like effects" $(p<.01)$ and "best" $(p<.001)$. In all three alcohol conditions, subjects on $50 \mathrm{mg}$ naltrexone reported significantly lower best and like effects compared to responses in the placebo naltrexone condition. There was an interaction of the high doses of naltrexone and alcohol on the two subjective measures of "best I've ever felt" $(p<.01)$ and "like effects of capsule/beverage" $(p<.05)$. Specifically, the combination of naltrexone $100 \mathrm{mg}$ and the high alcohol dose decreased ratings on these response items more than expected based on the separate effects of either drug. As shown in Figure 1A, naltrexone $50 \mathrm{mg}$ lowered reports of liking by $13 \%$ and naltrexone $100 \mathrm{mg}$ lowered reports of liking by $25 \%$ across the entire post-ingestion period. Reports of best effects were dampened $5 \%$ and $23 \%$ by the moderate and high naltrexone doses, respectively (Figure 1B). Interestingly, the temporal pattern of response dampening was somewhat different for best and like effects (Figure 1). In the $100 \mathrm{mg}$ naltrexone condition, the maximal reduction of liking was approximately $50 \%$ and occurred in the second half of the 8-hour session. In contrast, the maximal reduction in best effects occured early after drink ingestion during the first two hours of the eight hour session.

Sick/Unpleasant Effects. At baseline prior to alcohol administration, reports of nausea were modestly decreased in the two active naltrexone conditions relative to placebo naltrexone (both $p=.05$ ). Alcohol administration produced dose-dependent increases in unpleasant effects of alcohol, including uncomfortable, nauseated, and worst I've ever felt (Table 2). These unpleasant effects decreased gradually across the postingestion period (all time effects, $p<.001$ ). There was a significant effect of baseline ratings of nausea on ratings following alcohol administration $(p<.001)$, such that the higher a subject's baseline report, the higher the subject's post-ingestion rating of nausea.

As shown in Figure 2, alcohol administration induced higher levels of nausea when subjects were maintained on active compared to placebo naltrexone. Following the moderate alcohol dose (Figure 2A), selfreports of nausea averaged across the post-ingestion period were unchanged for subjects on $50 \mathrm{mg}$ naltrexone and increased $14 \%$ for subjects on $100 \mathrm{mg}$ naltrexone compared to placebo naltrexone. Nausea ratings following the high alcohol dose (Figure 2B) were increased $22 \%$ and $7 \%$ for subjects on $50 \mathrm{mg}$ and $100 \mathrm{mg}$ naltrexone, respectively. These subjective ratings of sick effects were paralleled by increased rates of vomiting following alcohol administration when subjects were on active naltrexone. There were eight episodes of vomiting during sessions, seven during alcohol sessions when subjects were on active naltrexone and one on placebo naltrexone $(Z=-3.21, p<.001)$. Generally, the vomiting occured well after the drink administration period, around two to three hours post-ingestion.

Sedative Effects. Alcohol administration increased a variety of sedative response items (Table 2). For all measures except sleepy, alcohol effects on sedation decreased gradually across the post-ingestion period (all time effects, $p<.001$ ). Scores on the PCAG (sedative) scale of the Addiction Research Center Inventory also were significantly increased following administration of moderate and high doses of alcohol.

Generally, sedative effects were increased in the active compared to placebo naltrexone conditions. These sedative effects of naltrexone were observed on a greater number of response items when subjects were maintained on the high compared to moderate dose of naltrexone (Table 2). However, significant interactions of high dose naltrexone and alcohol were observed on the sedative response items of floating, time distortion and PCAG scale scores such that subjects reported less sedation following the combination of alcohol and high dose naltrexone than would have been predicted by their separate effects.

High/Intoxication. Alcohol ingestion significantly increased self-reports of intoxication, including high, drunk and feel effects of alcohol or drugs (Table 2). These effects decreased gradually across the post-ingestion period (all time effects, $p<.001$ ). Naltrexone did not alter these measures of alcohol-related intoxication.

\section{Psychomotor Effects}

Stance Stability. In the absence of alcohol, there was a small but significant decrease in stance stability/left foot when subjects were maintained on the $50 \mathrm{mg}$ compared with placebo naltrexone $(p<.01)$. In general, alcohol ingestion impaired stance stability (Table 3). There were no systematic effects of naltrexone on stance stability following alcohol ingestion.

Reaction Time. In the absence of alcohol, there was a small but significant decrement in circular lights performance when subjects were maintained on $100 \mathrm{mg}$ (Mean lights extinguished $=106.6$ ) compared to placebo naltrexone (Mean lights extinguished $=109.4$ ) $(p=.01)$.

Circular lights performance was significantly decreased by the moderate and high dose of alcohol (Table 3). As shown in Figure 3, there were significant effects of $50 \mathrm{mg}$ and $100 \mathrm{mg}$ naltrexone on reaction time as measured by circular lights performance, with 100 $\mathrm{mg}$ naltrexone producing significantly greater impairment than $50 \mathrm{mg}$ naltrexone (Table 3). This effect of nal- 

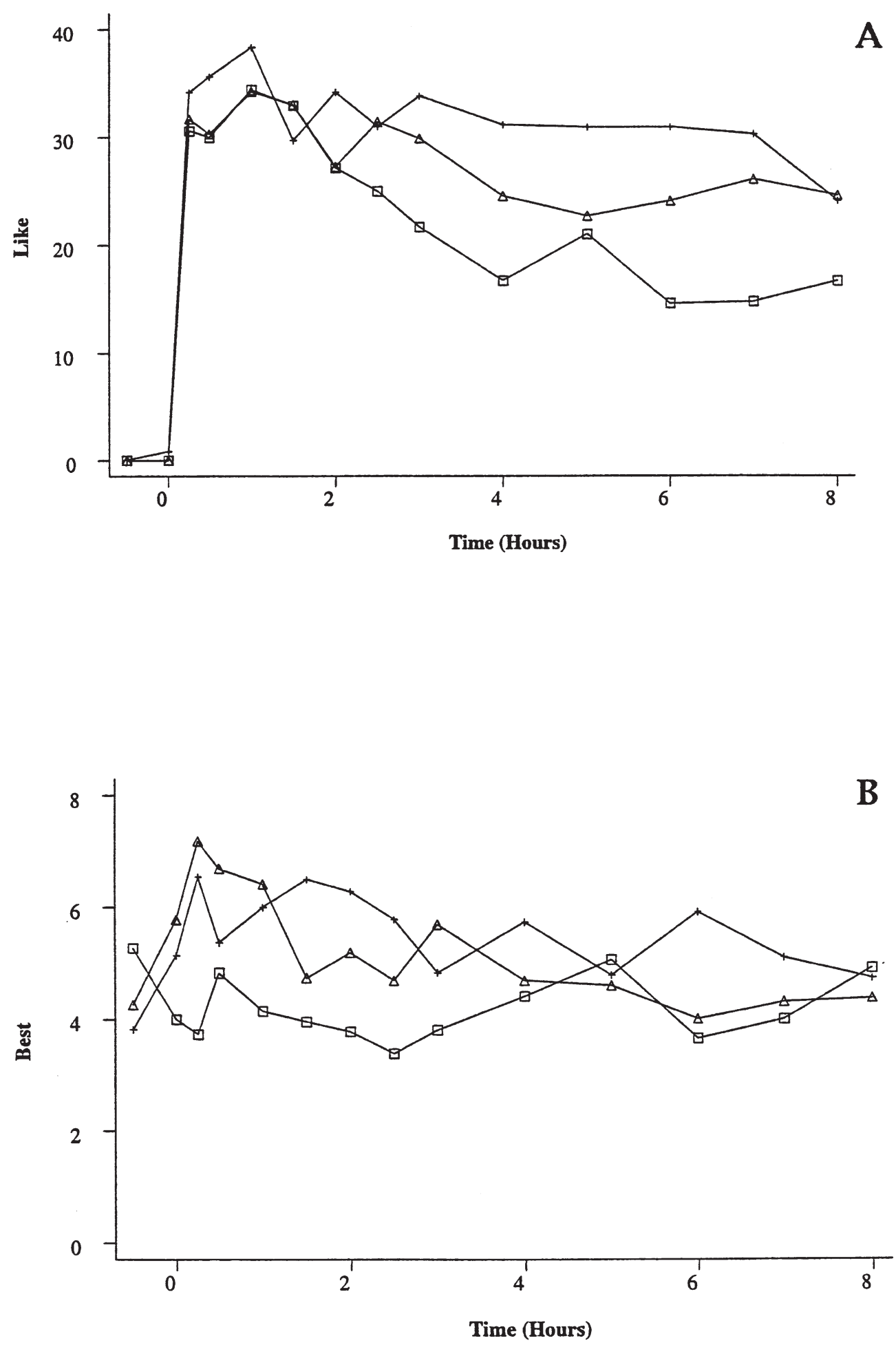

Figure 1. Analog scores for "liking of the capsule/beverage" (A) and "best I've ever felt" (B) prior to and following the high alcohol dose as a function of naltrexone dose condition. Symbols indicate means across subjects in each dose condition at each time point. Symbols to the left of the 0 time point represent baseline scores prior to alcohol administration. Naltrexone doses include placebo $(+), 50 \mathrm{mg}(\Delta)$, and $100 \mathrm{mg}(\square)$. Liking was scored on an analog scale from 0 ("not at all") to 39 ("extremely"). "Best I've ever felt" was scored on an analog scale from 0 ("normal") to 9 ("extremely"). 

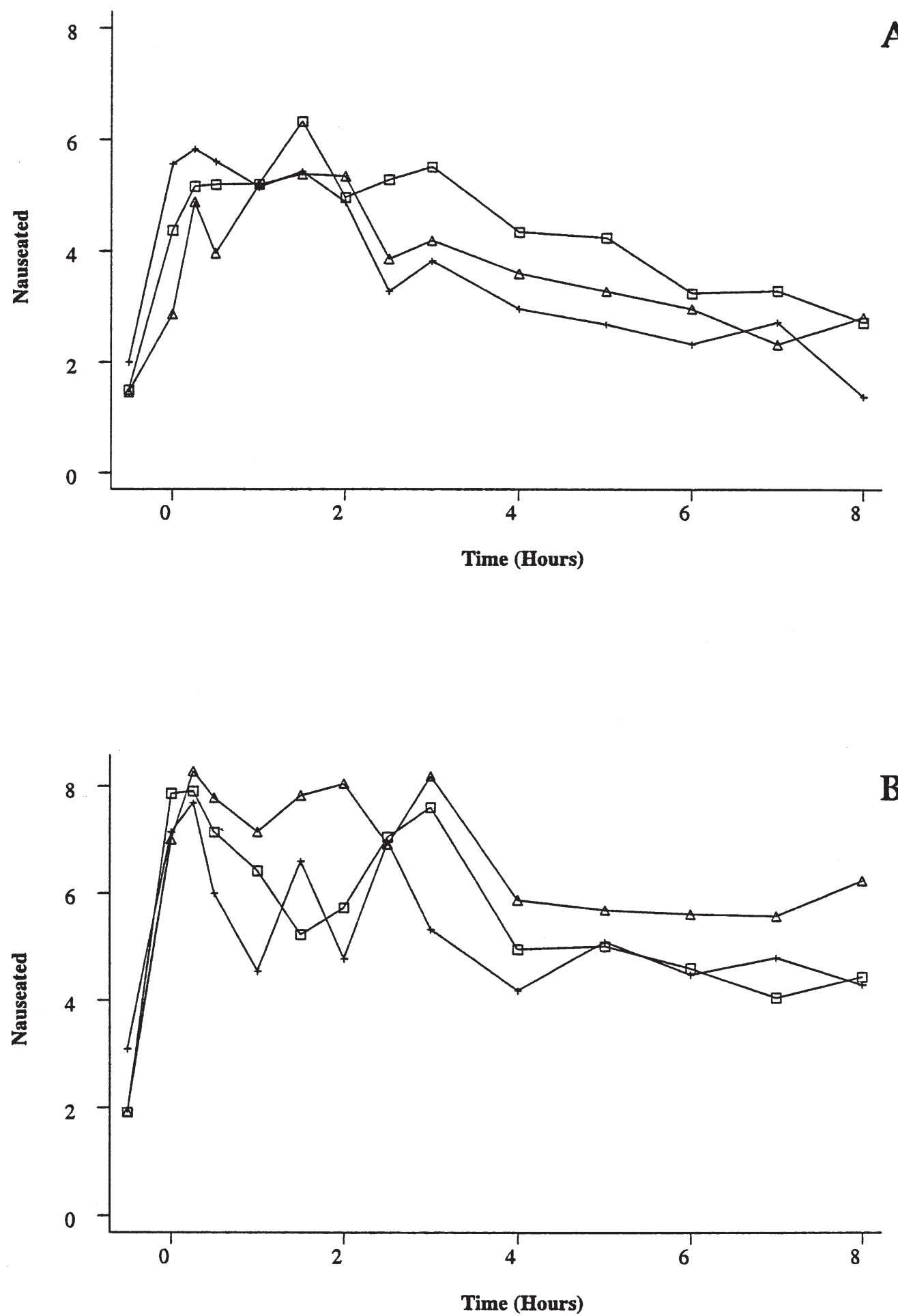

Figure 2. Analog scores for nauseated prior to and following the moderate (A) and high alcohol dose (B) as a function of naltrexone dose condition. Symbols indicate means across subjects in each dose condition at each time point. Symbols to the left of the 0 time point represent baseline scores prior to alcohol administration. Naltrexone doses include placebo $(+)$, 50 mg $(\Delta)$, and $100 \mathrm{mg}(\square)$. Nauseated was scored on an analog scale from 0 ("normal") to 9 ("extremely").

trexone on circular lights performance was primarily observed following the moderate dose of alcohol (Figure 3A). Across conditions, performance recovered over the 8 hour post-ingestion period (time; $p<.0001$ ).
Memory. At baseline in the absence of alcohol, there were no effects of naltrexone on memory as measured by DSST and recall performance. Ingestion of the high alcohol dose produced significant decrements in the number 
Table 3. Separate and Interactive Effects of Naltrexone and Alcohol on Psychomotor Performance. Arrows Indicate the Direction of Effect

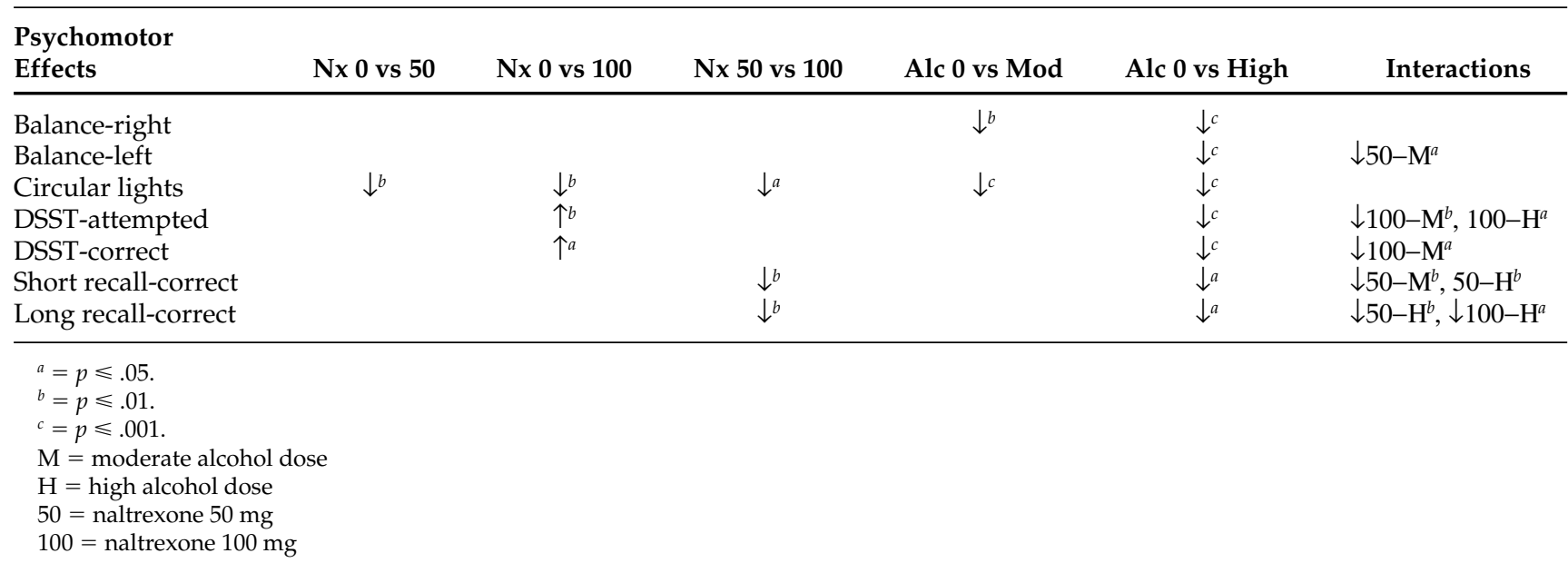

of attempted and correct DSST responses, with a trend towards lower numeric recall scores $(p=.02)$ (Table 3).

As shown in Table 3, there was significant improvement in DSST performance when subjects received 100 $\mathrm{mg}$ naltrexone. This effect appeared to be largely the result of improved performance for subjects on $100 \mathrm{mg}$ naltrexone in the placebo alcohol condition. Specifically, following placebo alcohol administration, subjects on $100 \mathrm{mg}$ naltrexone attempted significantly more DSST items $(p=.007)$ and tended to get more items correct $(p=.03)$ compared to placebo naltrexone. This improvement in performance did not occur when subjects on $100 \mathrm{mg}$ naltrexone received active alcohol. Following active alcohol administration, DSST performance was comparable across the naltrexone dose condition. Thus, there was a significant interaction of naltrexone and alcohol.

There also were interactions between naltrexone and alcohol on the short and long recall tasks, with the drug combination producing significantly greater impairment than the separate effects of the drugs. For no-delay numeric recall scores, there was an interaction of naltrexone $50 \mathrm{mg}$ with moderate and high dose alcohol. For delayed numeric recall scores, naltrexone $50 \mathrm{mg}$ and naltrexone $100 \mathrm{mg}$ in combination with high dose alcohol produced significant decrements in performance. In contrast to DSST performance, these interactions reflected greater decrements in performance following the combination of active alcohol and naltrexone rather than naltrexone-induced improvement in performance in the absence of active alcohol.

\section{Blood Alcohol Levels}

In the moderate alcohol dose condition, blood alcohol level peaked 30 minutes following ingestion at a mean of $0.048 \mathrm{mg} / \mathrm{dL}$; blood alcohol level averaged across the moderate alcohol dose sessions was 0.034 $\mathrm{mg} / \mathrm{dL}($ S.E. $=.033)$. In the high alcohol dose condition, blood alcohol level peaked 60 minutes following ingestion at a mean of $0.104 \mathrm{mg} / \mathrm{dL}$; mean blood alcohol level across the high alcohol dose sessions was $.088 \mathrm{mg} / \mathrm{dL}($ S.E. $=.043)$. There were no differences in mean or peak blood alcohol levels or time to peak BALs as a function of naltrexone dose condition (all $p>.10)$.

\section{DISCUSSION}

The present study findings provide strong support for the involvement of the endogenous opioid system in alcohol respones in that naltrexone administration altered a variety of subjective and objective measures of alcohol effects. In the present study, naltrexone decreased subjects' ratings of desire to drink both prior to and following alcohol administration. In approximately half of the subjects, this reduced urge for alcohol during naltrexone maintenance was paralleled by changes in several objective indicators of desire for alcohol including increased drink refusal and slower rates of drinking. Further, selfreports of "best I've ever felt" and "like effects of capsule/drink" were decreased across alcohol conditions among subjects receiving $50 \mathrm{mg}$ compared to placebo naltrexone. Importantly, there was a significant interaction between alcohol and naltrexone such that naltrexone $100 \mathrm{mg}$ in combination with the high alcohol dose produced greater decreases in ratings of "best I've ever felt" and "like effects of capsule/drink" than those obtained for each drug separately. Naltrexone also increased sedative and sick/unpleasant effects following alcohol ingestion. Subjective ratings of increased sick/unpleasant effects were consistent with the increased nausea and 

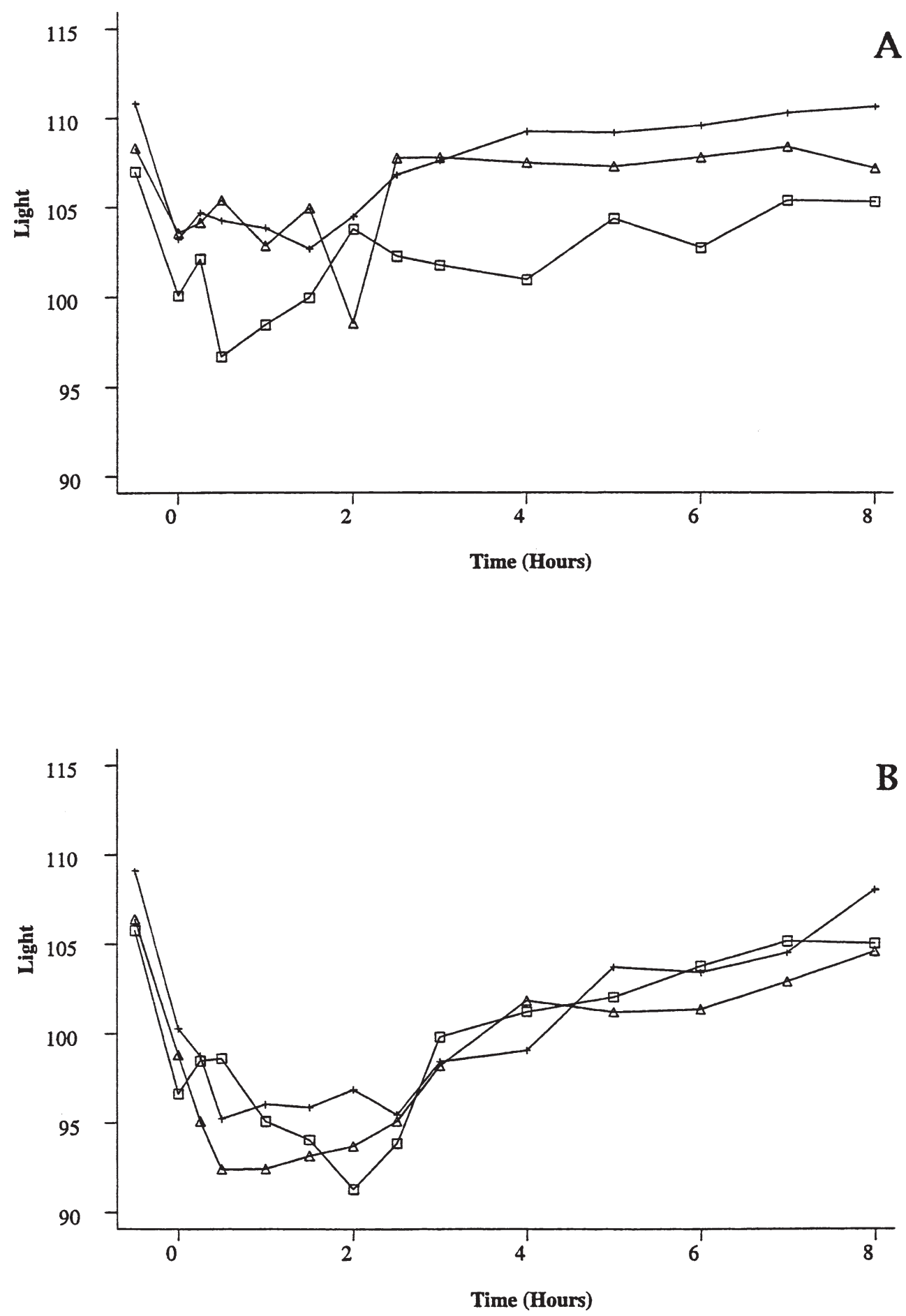

Figure 3. Circular lights performance prior to and following the moderate (A) and high alcohol dose (B) as a function of naltrexone dose condition. Symbols indicate means across subjects in each dose condition at each time point. Symbols to the left of the 0 time point represent baseline scores prior to alcohol administration. Naltrexone doses include placebo $(+), 50 \mathrm{mg}(\Delta)$, and $100 \mathrm{mg}(\square)$. Scores represent the number of lights that subjects extinguished in a 60-second period.

vomiting observed following alcohol ingestion during active compared to placebo naltrexone sessions.

It is notable that subjects' ratings of alcohol intoxication (high and drunk) were not altered by naltrex- one. This finding is in agreement with the comparable mean, peak and time to peak blood alcohol levels observed across naltrexone conditions, despite some disruption of drinking behaviors by naltrexone. 
Also, the objective psychomotor measures generally confirmed the similar subjective ratings of intoxication during active and placebo naltrexone sessions. Naltrexone produced systematic impairment only on circular lights performance. Results on the remaining psychomotor tasks were inconsistent and the actual magnitude of these effects was fairly small.

The results of the present study support and extend findings from earlier laboratory studies of naltrexone's effects on alcohol responses. Our findings of decreased liking and increased sedative effects associated with active naltrexone support the earlier observations by Swift et al. (1994), although no change in sedative effects was observed in two recent studies (Davidson et al. 1996; King et al. 1997). For example, Doty and colleagues (1997) failed to observe effects of naltrexone (25 or $50 \mathrm{mg}$ ) on stimulant or sedative responses to alcohol ingestion; however, their findings may be limited by several study design considerations including use of light-to-moderate social drinkers as study subjects, acute administration of the naltrexone dose one hour before alcohol ingestion, and selection of a very low alcohol dose (0.25) $\mathrm{g} / \mathrm{kg}$ that produced only mild subjective effects. Our findings of somewhat limited effects on psychomotor responses agree with earlier reports of no significant effects of naltrexone compared to placebo on alcohol-related decrements in reaction time and memory tasks (Swift et al. 1994). Of particular interest, there was no indication of increased nausea at baseline among subjects receiving active naltrexone. Additionally, our observation of increased self-reports of nausea and unpleasant/sick effects as well as the increased likelihood of vomiting following active but not placebo alcohol refutes suggestions that decreased alcohol consumption is a non-specific effect of naltrexone associated with general nausea or unpleasant subjective effects in the absence of alcohol consumption. This observation of naltrexone-precipitated nausea only in the presence of active alcohol is in line with earlier reports (Davidson et al. 1996; King et al. 1997; Swift et al. 1994). Our observations of increased drink refusal and delay in drink completion extend the recent report of delays in initiating drinking among subjects on $50 \mathrm{mg}$ naltrexone (Davidson et al. 1996), although Davidson and colleagues did not find differences in number of drinks requested or time to drink completion. Finally, none of the laboratory studies conducted to date including the present report have observed differences in alcohol pharmacokinetic parameters including peak, time to peak and area under the curve as a function of naltrexone dose (King et al. 1997; Swift et al. 1994).

Results of the present study also are informative with respect to observations in the earlier clinical trials with alcohol-dependent patients. As was observed by Volpicelli and colleagues (1992), subjects in the present study who received $50 \mathrm{mg}$ compared to placebo naltrexone reported reduced desire to drink at baseline in the absence of alcohol; a similar trend of decreased craving was observed for subjects on $100 \mathrm{mg}$ naltrexone. Importantly, the present study has extended these findings on craving to include the post-alcohol ingestion period. Our findings indicate that subjects receiving $100 \mathrm{mg}$ naltrexone also report decreased desire to drink following alcohol ingestion. Increased drink refusal and a slower pace to drink completion also were observed and could be interpreted as objective indicators of these self-reported changes in desire to drink. These findings are noteworthy since, across the published clinical trials, naltrexone appeared to differentially affect risk of return to heavy drinking but not risk of any drinking. That is, naltrexone- and placebotreated subjects were equally likely to ingest an alcohol drink, but naltrexone-treated subjects were significantly less likely to continue drinking to alcohol relapse. Our findings of nausea only following active alcohol ingestion suggest that some of the risk of increased nausea associated with naltrexone ingestion in the earlier clinical trials (Croop et al. 1997) may be related to subjects' drinking status and not a medication side-effect alone. Finally, in the earlier clinical trial by Volpicelli and colleagues, naltrexone-treated subjects who did drink reported less "high" than placebo-treated subjects who drank, although no differences were observed in reported intoxication, craving, or loss of temper (Volpicelli et al. 1995). In the present study, self-reported high following alcohol ingestion was not decreased in either naltrexone dose condition. This difference may stem from a greater range of descriptive terms available to characterize alcohol effects in the current study, allowing a greater refinement in subjects' responses. Alternatively, as the authors suggest, it may indicate that earlier findings were a result of reduced alcohol consumption by naltrexone versus placebo subjects (Volpicelli et al. 1995).

The present study has several design strengths. First, subjects were housed on an inpatient research unit throughout each naltrexone dosing cycle. We were able to observe naltrexone ingestion and can rule out medication noncompliance as a factor in the study findings. Inpatient hospitalization also minimized the risk of unauthorized alcohol ingestion or drug use during the active dosing periods. Second, the study used a withinsubject design, crossing all alcohol and naltrexone doses for each subject. This design increased the statistical power of our study to observe main and interaction effects of naltrexone and alcohol, given that such human laboratory studies often have relatively small sample sizes. Finally, we selected a chronic dosing model for naltrexone administration. This procedure more closely parallels naltrexone's use in clinical populations, increases the likelihood that naltrexone and b-nal- 
trexol (the major metabolite of naltrexone) are at steady-state across alcohol dose conditions, and decreases the risk of acute, naltrexone-induced nausea interfering with the study measures.

Despite these design strengths, there also were two potential limitations to the current study. First, the study sample consisted of heavy drinkers rather than alcohol-dependent subjects. At present, there is a lack of consensus on the most appropriate population for laboratory studies involving alcohol administration. Current Institutional Review Board and National Institute on Alcohol Abuse and Alcoholism guidelines discourage the conduct of alcohol challenge studies in alcoholdependent persons. Further, conducting laboratory studies in alcohol-dependent persons who may be undergoing alcohol withdrawal clearly creates another set of interpretative problems. Because of these concerns, the heavy drinking population seems to be a reasonable surrogate for alcohol-dependent subjects. The fact that our laboratory results parallel many of the clinical trial findings for naltrexone in alcohol dependent subjects provides face validity to the subject selection used in this study. Also, the growing clinical interest in prophylactic use of naltrexone for management of heavy drinking in non-alcohol dependent persons increases the direct clinical relevance of the current findings. Second, almost half of the subjects acknowledged marijuana use and one-quarter reported occasional cocaine use; it is not known how this other drug use may influence naltrexone's effects on alcohol responses. Although subjects were instructed to report for each inpatient admission alcohol and drug free, there was no fool-proof method to completely eliminate substance use. Several procedures were put into place to minimize the potential impact of any substance use: subjects underwent a 3-day inpatient stay before alcohol dosing procedures began; urine screens and breathalyzer results were obtained at each inpatient admission and randomly throughout patients' 8-day inpatient stays. Two subjects were excluded as a result of urinalysis evidence of drug use during the GCRC stay. Thus, we can be reasonably confident that current study findings reflect the interaction of naltrexone and alcohol only and not the interaction with other drugs.

A primary purpose of this study was to examine the interactions of naltrexone and alcohol dosage levels in an effort to address the practical issue of optimal naltrexone dosing for clinical effectiveness. In examining the main effects of $50 \mathrm{mg}$ and $100 \mathrm{mg}$ naltrexone, there was in general little evidence of a naltrexone dose-effect function. Specifically, both doses increased sedative and unpleasant/sick effects following alcohol administration, and neither dose decreased self-reports of intoxication. However, although naltrexone $50 \mathrm{mg}$ was more effective in decreasing baseline reports of desire to drink, only the high naltrexone dose condition reduced craving following alcohol administration. Perhaps most importantly, the high dose of naltrexone was especially effective in decreasing reported well-being and liking of effects after subjects had consumed the high dose of alcohol. These findings would seem to suggest that optimal naltrexone dosing may be best determined by a patient's risk for heavy drinking during treatment.

Our findings highlight the importance of including a range of doses in research examining naltrexone effects on alcohol responses, since interactions can occur at dose combinations that would be missed if only a single dose of either drug were studied. Also, the present study results highlight several key areas for further examination in laboratory and clinical trials research. Specifically, it will be important to examine closely the timing and context of reports of naltrexone-precipitated nausea to sort out true medication side-effects from naltrexone-alcohol interactions. Also, naltrexone's effects on craving both in the presence and absence of alcohol should be followed up to better understand the role that these play in helping to prevent relapse in recently abstinent alcoholics. Finally, if desire to drink, best and liking are indeed opioid modulated effects of alcohol, the ability of naltrexone to attenuate these responses continues to support the premise that naltrexone's effects are mediated via blockade of opiate receptors. Further research is still needed to fully understand these mechanisms of naltrexone's actions.

\section{ACKNOWLEDGMENTS}

A portion of this work was presented at the 1997 meeting of the Research Society on Alcoholism. The study was supported by USPHS grant R01-AA09569 and Johns Hopkins Bayview Medical Center General Clinical Research Center grant M01RR02719. The authors thank Joseph Bezold, Shing Lee, and John Yingling for their help in conducting this research and analyzing the data.

\section{REFERENCES}

Altshuler HL, Phillips PE, Feinhandler DA (1980): Alteration of ethanol self-administration by naltrexone. Life Sciences 26:679-688

Bigelow GE, and Walsh SL (1998): Evaluation of potential pharmacotherapies: Response to cocaine challenge in the human laboratory. In Higgins, ST, Katz, JL (eds), Cocaine Abuse: Behavior, Pharmacology, and Clinical Applications. California, Academic Press, pp 209-238

Boyle AEL, Stewart RB, Macenski MJ, Spiga R, Johnson BA, Meisch RA (1998): Effects of acute and chronic doses of naltrexone on ethanol self-administration in rhesus monkeys. Alcohol Clin Exp Res 22:359-366

Croop RS, Faulkner EB, Labriola DF, for The Naltrexone Usage Study Group (1997): The safety profile of naltrex- 
one in the treatment of alcoholism. Archives General Psychiatry 54:1130-1135

Czirr SA, Hubbell CL, Reid LD (1987): Daily water deprivation potentiates ethanol intake beyond a period of water intake. Alcohol 4:117-120

Davidson D, Amit Z (1997): Effect of ethanol drinking and naltrexone on subsequent drinking in rats. Alcohol 14:581-584

Davidson D, Swift R, Fitz E (1996): Naltrexone increases the latency to drink alcohol in social drinkers. Alcohol Clin Exp Res 20:732-739

Doty P, de Wit H (1995): Effects of naltrexone pretreatment on the subjective and performance effects of ethanol in social drinkers. Behav. Pharmacol 6:386-394

Doty P, Kirk JM, Cramblett MJ, de Wit H (1997): Behavioral responses to ethanol in light and moderate social drinkers following naltrexone pretreatment. Drug and Alcohol Dependence 47:109-116

Froehlich JC, Harts J, Lumeng L, Li TK (1990): Naloxone attenuates voluntary ethanol intake in rats selectively bred for high ethanol preference. Pharmacology, Biochemistry \& Behavior 35:385-390

Gianoulakis C (1990): Characterization of the effects of acute ethanol administration on the release of beta-endorphin peptides by the rat hypothalamus. European Journal of Pharmacology 180:21-29

Gianoulakis C, Krishnan B, Thavundayil J (1996): Enhanced sensitivity of pituitary beta-endorphin to ethanol in subjects at high risk of alcoholism. Archives of General Psychiatry 53:250-257

Haertzen CA (1974): An overview of Addiction Research Center Inventory scales (ARCI): An appendix and manual of scales. Vol. DHEW Publication No. (ADM) 74-92. National Institute on Drug Abuse, Rockville, MD

Hubbell CL, Czirr SA, Hunter GA, Beaman CM, LeCann NC, Reid LD (1986): Consumption of ethanol solution is potentiated by morphine and attenuated by naloxone persistently across repeated daily administrations. Alcohol 3:39-54

Hubbell CL, Marglin SH, Spitalnic SJ, Abelson ML, Wild KD, Reid LD (1991): Opioidergic, serotonergic, and dopaminergic manipulations and rats' intake of a sweetened alcoholic beverage. Alcohol 8:355-367

Hyytia P, Sinclair JD (1993): Responding for oral ethanol after naloxone treatment by alcohol-preferring AA rats. Alcohol Exp Clin Res 17:631-636

King AC, Volpicelli JR, Frazer A, O’Brien CP (1997): Effect of naltrexone on subjective alcohol response in subjects at high and low risk for future alcohol dependence. Psychopharmacology 129:15-22

Krishnan-Sarin S, Portoghese PS, Li TK, Froehlich JC (1995): The delta opioid receptor antagonist naltriben selectively attenuates alcohol intake in rats bred for alcohol preference. Pharmacology, Biochemistry \& Behavior 52:153-159

Li XW, Li TK, Froehlich JC (1996): Alcohol alters preproenkephalin mRNA content in the shell and core of the nucleus accumbens. Alcoholism: Clinical and Experimental Research 20:53

McCaul ME, Turkkan JS, Svikis DS, Bigelow GE (1990): Alco- hol and secobarbital effects as a function of familial alcoholism: Acute psychophysiological effects. Alcoholism: Clinical and Experimental Research 14:704-712

McLeod DR, Griffiths RR, Bigelow GE, Yingling J (1982): An automated version of the digit symbol substitution task (DSST). Behavior Research, Methods \& Instrumentation 14:463-466

Myers RD, Borg S, Mossberg R (1986): Antagonism by naltrexone of voluntary alcohol selection in the chronically drinking macaque monkey. Alcohol 3:383-388

O'Malley SS, Jaffe AJ, Chang G, Schottenfeld RS, Meyer RE, Rounsaville B (1992): Naltrexone and coping skills therapy for alcohol dependence. A controlled study. Archives of General Psychiatry 49:881-887

O'Malley SS, Jaffe AJ, Rode S, Rounsaville BJ (1996): Experience of a "slip" among alcoholics treated with naltrexone or placebo. American Journal of Psychiatry 153: 281-283

Phillips TJ, Wenger CD, Dorow JD (1997): Naltrexone effects on ethanol drinking acquisition and on established ethanol consumption in C57BL/6J mice. Alcohol Clin Exp Res 21:691-702

Reid LD, Delconte JD, Nichols ML, Bilsky EJ, Hubbell CL (1991): Tests of opioid deficiency hypotheses of alcoholism. Alcohol 8:247-257

Reid LD, Gardell LR, Chattopadhyay S, Hubbell CL (1996): Periodic naltrexone and propensity to take alcoholic beverage. Alcohol Clin Exp Res 20:1329-1334

Schuckit MA (1980): Self-rating of alcohol intoxication by young men with and without family histories of alcoholism. Journal of Studies on Alcohol 41:242-249

Selzer ML (1971): The Michigan Alcoholism Screening Test: The quest for a new diagnostic instrument. American Journal of Psychiatry 127:1653-1658

Spitzer RL, Williams JB W (1987): Structured Interview for DSM-IIIR (SCID). New York State Psychiatric Institute, Biometrics Research, New York

Swift RM, Whelihan W, Kuznetsov O (1994): Naltrexoneinduced alterations in human ethanol intoxication. American Journal of Psychiatry 151:1463-1467

Volpicelli J, David MA, Olgin JE (1986): Naltrexone blocks the post-shock increase of ethanol consumption. Life Sciences 38:841-847

Volpicelli JR, Alterman AI, Hayashida M, O’Brien CP (1992): Naltrexone in the treatment of alcohol dependence. Archives of General Psychiatry 49:876-880

Volpicelli JR, Watson NT, King A, Sherman CE, O’Brien CP (1995): Effect of naltrexone on alcohol "high" in alcoholics. Am J Psychiatry 152:613-615

Wand GS, Mangold D, El Diery S, McCaul ME, Hoover D (1998): Offspring from families with a high density of alcohol dependent individuals have altered endogenous brain opioidergic activity. Archives of General Psychiatry 55:1114-1119

Weiss F, Mitchiner M, Bloom FE, Koob GF (1990): Freechoice responding for ethanol versus water in alcohol preferring $(\mathrm{P})$ and unselected Wistar rats is differentially modified by naloxone, bromocriptine, and methysergide. Psychopharmacology 101:178-186 